\title{
Phase reciprocity of spin-wave excitation by a microstrip antenna
}

\author{
T. Schneider, * A. A. Serga, T. Neumann, and B. Hillebrands \\ Fachbereich Physik, Technische Universität Kaiserslautern, 67663 Kaiserslautern, Germany \\ M. P. Kostylev ${ }^{\dagger}$ \\ School of Physics, University of Western Australia, Crawley, Western Australia 6009, Australia
}

(Received 8 April 2008; published 9 June 2008)

\begin{abstract}
Using space-, time-, and phase-resolved Brillouin light-scattering spectroscopy we investigate the difference in phase of the two counterpropagating spin waves excited by the same microwave microstrip transducer. These studies are performed both for backward volume magnetostatic waves and magnetostatic surface waves in an in-plane magnetized yttrium iron garnet film. The experiments show that for the backward volume magnetostatic spin waves (which are reciprocal and excited symmetrically in amplitude) there is a phase difference of $\pi$ associated with the excitation process and thus the phase symmetry is distorted. On the contrary, for the magnetostatic surface spin waves (which are nonreciprocal and unsymmetrical in amplitude) the phase symmetry is preserved (there is no phase difference between the two waves associated with the excitation). Theoretical analysis confirms this effect.
\end{abstract}

DOI: 10.1103/PhysRevB.77.214411

PACS number(s): 75.30.Ds, 78.35.+c

\section{INTRODUCTION}

It is well-known that two very different kinds of dipolar dominated spin waves can propagate in an in-plane magnetized magnetic film. ${ }^{1}$ One of them, the so-called backward volume magnetostatic spin wave (BVMSW) (magnetic bias field in the film plane and parallel to the propagation direction), is characterized by a harmonic distribution of the dynamic magnetization across the film thickness and is completely reciprocal. The other one, the magnetostatic surface spin wave (MSSW) (magnetic bias field is in-plane, but perpendicular to the propagation direction), has an exponential distribution of the dynamic magnetization on the film thickness. When the spin-wave propagation direction is reversed the maximum of the dynamic magnetization of this mode shifts from one film surface to the other. The surface wave is thus nonreciprocal. Influence of the reciprocity is important for many spin-wave phenomena connected both with parametric $^{2,3}$ and nonlinear ${ }^{4,5}$ interactions of counterpropagating waves, including reflection ${ }^{6}$ and excitation. ${ }^{7}$

One of the most evident manifestations of this influence is that the amplitudes of two counterpropagating spin waves excited by a microwave current through an antenna mounted on top of the magnetic film significantly depend on the used geometry. In the case of reciprocal BVMSWs these waves propagate with identical amplitudes. If one excites the nonreciprocal MSSWs this amplitude symmetry is drastically distorted and spin-wave propagation in one direction is strongly suppressed. This amplitude effect is immediately visible and thus always taken into account in experiments on excitation of spin waves by microstrip transducers placed near the film surfaces.

In contrast to the spin-wave amplitude the reciprocity of the phase of spin waves excited that way is not obvious. In this paper we demonstrate that the phase symmetry is distorted when the reciprocal BVMSW is excited and is preserved in the case of the nonreciprocal MSSW. As a measure of the phase symmetry we used the difference in initial phases of the two counterpropagating waves excited by the same transducer. In the following, we will term this phase difference as the "excitation phase."

The importance of the excitation phase is clear in all cases when linear interference or nonlinear interaction of the excited counterpropagating waves is possible. This is the usual situation for rectangular and especially for ring resonators (see e.g., Refs. 8 and 9). The influence of the excitation phase has also been pointed out in the previous works on spin-wave soliton collision ${ }^{4,5}$ and on nonlinear pattern formation. ${ }^{10}$ In particular, in Ref. 10 the excitation phase determines the selection rule for excitation of nonlinear standing-wave spin-wave resonances in a rectangular monodomain magnetic square, which is important from the point of view of switching elements of magnetic memory devices. Knowledge of the initial phase of spin waves is also important for recently suggested spin-wave logic applications. ${ }^{11-13}$

The conventional method of spin-wave detection using for example two microstrip antennae equally spaced on both sides of the input transducer cannot be used to measure the excitation phase due to the symmetry of the excitation and detection processes. Detecting the spin waves using this technique would lead to an additional phase shift of the same magnitude than the excitation phase but of the opposite sign. The microwave signals in the output antennae will thus always be in phase, regardless of the initial phase difference between the two spin waves. This problem is general for all situations when detection is performed via inducted microwave currents. It can now be avoided due to the recent development of phase-resolved Brillouin light-scattering (BLS) spectroscopy technique, ${ }^{14}$ an extension of the space- and time-resolved BLS, ${ }^{15}$ and the possibility to observe variation of spin-wave phase with propagation distance (the so-called phase accumulation ${ }^{16}$ ), which allows us to measure the excitation phase of microwave excited spin waves using a purely optical technique.

Using the described method we were able to directly observe the difference in excitation phase between the BVMSW and MSSW geometry. We also suggest a theoretical explanation for the observed effect. 


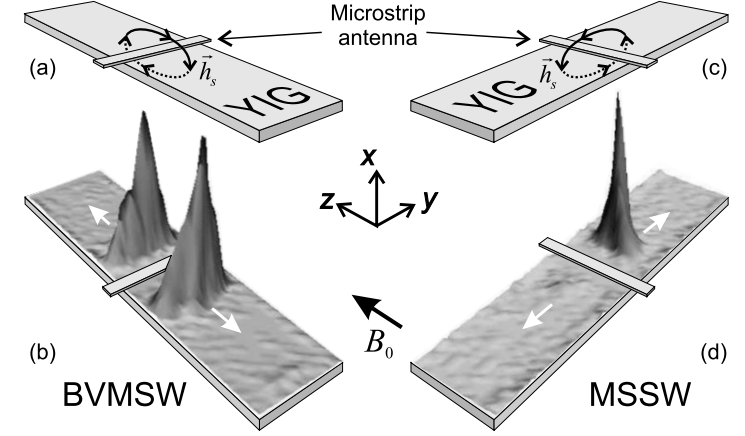

FIG. 1. (a) Experimental setup for BVMSW geometry. Spin waves were excited using a microstrip antenna. (b) Space resolved BLS measurement of the intensity of two counterpropagating spin waves in the BVMSW geometry approximately $40 \mathrm{~ns}$ after their excitation. (c) Experimental setup for MSSW geometry. (d) Space resolved BLS measurement of two counterpropagating spin waves in the MSSW geometry approximately $40 \mathrm{~ns}$ after their excitation.

\section{RESULTS FOR THE BACKWARD VOLUME MAGNETOSTATIC SPIN-WAVE CONFIGURATION}

Figure 1(a) shows a picture of the experimental setup. Spin waves are excited using a microstrip antenna placed on top of a $5 \mu \mathrm{m}$ thick yttrium iron garnet (YIG) spin-wave waveguide. The antenna width $w$ is $50 \mu \mathrm{m}$. Spin waves excited by microwave pulses applied to the antenna can propagate freely in both directions, as demonstrated in Fig. 1(b).

A typical example of the BVMSW phase accumulation measurement performed in this work is shown in Fig. 2 [bias magnetic field 1835 Oe is applied in plane, parallel to the propagation direction, as shown in Fig. 1(a), carrier frequency is $7.132 \mathrm{GHz}$. One can clearly see a linear variation of the phase $\phi(z)$. As we have used a linear, quasimonochromatic wave, the accumulated phase can be described as wave vector $k$ times the propagation distance $|z|$ (plus the initial

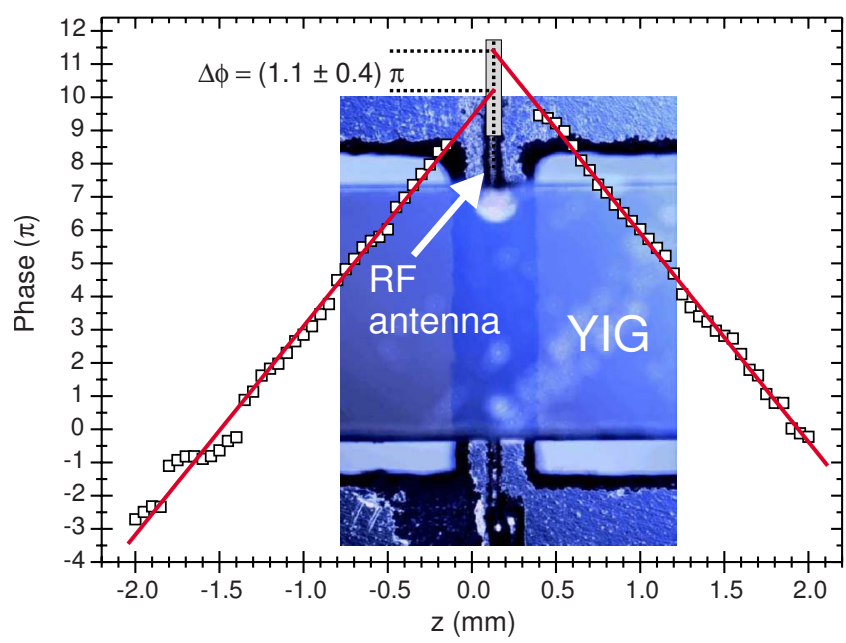

FIG. 2. (Color online) Phase accumulation for BVMSW geometry. The measured excitation phase is $(1.1 \pm 0.4) \pi$. The applied bias magnetic field is 1835 Oe. Spin-wave carrier frequency and wave vector are $7.132 \mathrm{GHz}$ and $(-198 \pm 1) \mathrm{cm}^{-1}$, respectively. The inset shows a micrograph of the used structure. phase). One sees that the phase decreases with propagation distance as is characteristic for a BVMSW mode.

Using a micrograph of the microstrip antenna and the substrate holding it (shown in the inset of Fig. 2) one can identify the position of the antenna in the measured data. The extrapolation of the linear fit of the phase accumulation up to the center of the antenna allows one to determine the excitation phase of the spin wave under investigation as $(1.1 \pm 0.4) \pi$ in the shown example. Other measurements performed for different bias magnetic fields (and thus different wave vectors) confirm a value of $\pi$ for the excitation phase of BVMSW within the error margins.

This result can be understood based on the configuration of the external magnetic field and the distribution of the dynamic magnetization associated with BVMSW excitation and propagation. Using the coordinate system defined in Fig. 1(a) one finds that the dynamic magnetization $\boldsymbol{m}$ lays in the $x-y$ plane, while the microwave field created by the antenna $\boldsymbol{h}_{s}$ has components in $x$ and $z$ directions only. The relation between the components of the effective dynamic field and the dynamic magnetization is given by the microwave susceptibility tensor $\hat{\chi}$ (see e.g., Ref. 17). This relation involves only the components perpendicular to the direction of the vector of static magnetization. In our case the components of the effective dynamic magnetic field which exert considerable torques onto the magnetization vector are the antenna microwave field $\boldsymbol{h}_{s}$ and the spin-wave dipole field $\boldsymbol{h}_{d}$. Therefore this relation takes the form

$$
\boldsymbol{m}=\hat{\chi} \cdot\left(\boldsymbol{h}_{s}+\boldsymbol{h}_{d}\right) .
$$

In the following we will consider the dynamic magnetization and the effective magnetic field averaged across the film thickness. In this approximation the dipole field of longwavelength spin waves is well described by the quasi-onedimensional tensorial Green's function $\hat{G}$ (see Ref. 18). This description is valid for $k L<1.5$ (where $k$ is the spin-wave wave vector and $L$ is the film thickness). ${ }^{19}$ The function has only two nonvanishing components, both of which are diagonal: the out-of-plane $\rightarrow$ out-of-plane component $G^{(o o)}$ and the in-plane $\rightarrow$ in-plane component $G^{(i i)}$. The dipolar field $\boldsymbol{h}_{d}$ can be written as

$$
\boldsymbol{h}_{d}(r)=\int_{-\infty}^{\infty} \hat{G}\left(r-r^{\prime}\right) \boldsymbol{m}\left(r^{\prime}\right) d r^{\prime},
$$

where $r$ is the coordinate along the spin-wave propagation path. It is important that both components are symmetric: $\hat{G}(-s)=\hat{G}(s)$. Therefore the dipole field does not introduce nonreciprocity into the propagation of long-wavelength spin waves.

As BVMSWs possess only an out-of-plane component of the dipolar field, Eq. (2) can be written as

$$
h_{d x}=\int_{-\infty}^{\infty} G^{(o o)}\left(r-r^{\prime}\right) m_{x}\left(r^{\prime}\right) d r^{\prime} .
$$

Furthermore, only the out-of-plane component of the antenna microwave field $\left(h_{s x}\right)$ lies in the plane perpendicular to the static magnetization. Under these conditions Eqs. (1) and 


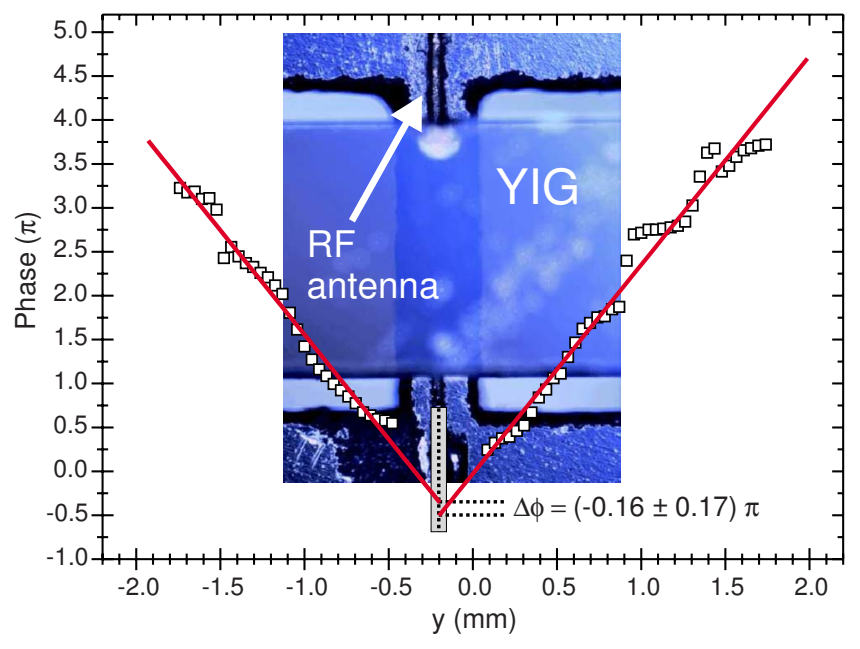

FIG. 3. (Color online) Phase accumulation for MSSW geometry. The measured excitation phase is $(-0.16 \pm 0.17) \pi$. The applied bias magnetic field is 1825 Oe. Spin-wave carrier frequency and wave vector are $7.132 \mathrm{GHz}$ and $(75 \pm 1) \mathrm{cm}^{-1}$, respectively. The inset shows a micrograph of the used structure.

(2) reduce to an inhomogeneous scalar integral equation derived in Ref. 20 that involves only $m_{x}, G^{(o o)}, h_{s x}$, and the diagonal component of $\hat{\chi}$. This results in equal amplitudes for the waves propagating in positive and negative direction. It also translates the symmetry of $h_{s x}(z)$ to the phase accumulation profile $\phi(z)$. Since $h_{s x}(z)$ has an antisymmetric profile along the $z$ axis with a node in the middle of the antenna this leads to an excitation phase of $\pi$.

Indeed, as follows from Eq. (B18) in Ref. 20, far away from the antenna $(|z| \gg w / 2)$ the expression for the dynamic magnetization takes the form

$$
m_{x}( \pm|z|)=\frac{4 \pi i}{L} h_{\mp|k| s x} \exp (i|k z|) .
$$

Note, that the sign in front of the complex exponent is positive since the waves are backward ones. This results in the observed phase accumulation $\phi(y)=-|k z|$. The magnitude $h_{k s x}$ is the spatial Fourier component of $h_{s x}(z)$ corresponding to the spatial frequency $k$. As mentioned before $h_{s x}(z)$ is an odd function of $z$ with a node in the middle of the antenna, so $h_{-|k| s x}=-h_{|k| s x}$, which gives the excitation phase of $\pi$ as confirmed in our measurements.

\section{RESULTS FOR THE MAGNETOSTATIC SURFACE SPIN-WAVE CONFIGURATION}

Figure 3 shows a typical example of the measurements for MSSW geometry (bias magnetic field 1825 Oe is applied in plane, perpendicular to the propagation direction, as shown in Fig. 1(c), carrier frequency is $7.132 \mathrm{GHz}$ ). As in the BVMSW case the phase accumulation $\phi(y)$ on both sides from the antenna is linear, but this time the phase increases with the propagation distance. The excitation phase is determined as $(-0.16 \pm 0.17) \pi$. Additional measurements for different bias magnetic fields confirm the zero value of MSSW excitation phase within the error margins.
Thus in the MSSW geometry the situation is quite different from the BVMSW case. This can be explained based on Eq. (1). As it is demonstrated in Fig. 1(c), for the MSSW case both fields $\boldsymbol{h}_{d}$ and $\boldsymbol{h}_{s}$ as well as the dynamic magnetization $\boldsymbol{m}$ lay in the $x-y$ plane. Therefore all components of the vectors $\boldsymbol{h}_{d}$ and $\boldsymbol{h}_{s}$ in Eq. (1) have to be taken into account. Contributions of all these components are efficiently intermixed by the microwave susceptibility tensor through the products $\hat{\chi} \cdot \boldsymbol{h}_{s}$ and $\hat{\chi} \cdot \boldsymbol{h}_{d}$. The result of intermixing is not trivial and can be space and wave vector dependent, because: (i) The antidiagonal components of the microwave susceptibility tensor are shifted in phase by $\pm \pi / 2$ with respect to the diagonal components; (ii) below the film surface the in-plane component of the antenna field $h_{s y}$ has the same phase everywhere, whereas the out-of-plane component $h_{s x}$ changes its phase by $\pi$ at the longitudinal antenna axis $y=0$; and (iii) the in-plane and the out-of-plane components of the Green's function are dependent on the spin-wave wave vector in different ways (see e.g., Ref. 21).

Thus the behavior of the complex amplitude of MSSWs can be nontrivial. A well-known manifestation of this behavior is the nonreciprocity of MSSW excitation by microstrip transducers. These waves propagate with significantly different amplitudes in the positive and negative directions from a microstrip antenna. This MSSW nonreciprocity is clearly seen in our BLS measurement shown in Fig. 1(d).

The system of Eqs. (1) and (2) for MSSWs allows for an analytical solution. As in the case of BVMSWs ${ }^{20}$ the solution involves a spatial Fourier transformation of the Green's function of the dipole field. An explicit formula relating the complex vector $\boldsymbol{m}$ to $\boldsymbol{h}_{s}$ can be found e.g., in Ref. 22. Here we obtain a solution in a form which allows for an expression for the excitation phase. Following the same method as in Ref. 20 from Eqs. (1) and (2) one arrives at the solution for the dynamic magnetization vector

$$
\boldsymbol{m}(y)=\int_{-\infty}^{+\infty} \hat{G}_{\mathrm{exc}}\left(y-y^{\prime}\right) \boldsymbol{h}_{s}\left(y^{\prime}\right) d y^{\prime},
$$

where

$$
\hat{G}_{\mathrm{exc}}\left(y-y^{\prime}\right)=\frac{1}{D(k)} \hat{Y} F\left(y-y^{\prime}\right),
$$

and the matrix $\hat{Y}$ has the elements

$$
\begin{gathered}
Y_{11}=-W(k)-1+\frac{\chi}{\left(\chi_{a}^{2}-\chi^{2}\right)}, \\
Y_{12}=\frac{i \chi_{a}}{\left(\chi_{a}^{2}-\chi^{2}\right)}, \\
Y_{21}=-\frac{i \chi_{a}}{\left(\chi_{a}^{2}-\chi^{2}\right)}, \\
Y_{22}=W(k)+\frac{\chi}{\left(\chi_{a}^{2}-\chi^{2}\right)},
\end{gathered}
$$

and 


$$
\begin{aligned}
F(s)= & \frac{1}{2 \pi}\left[-2 \pi i \exp \left(-i k_{c}|s|\right)+\exp \left(-i k_{c}^{*}|s|\right) E_{1}\left(-i k_{c}^{*}|s|\right)\right. \\
& \left.+\exp \left(i k_{c}^{*}|s|\right) E_{1}\left(i k_{c}^{*}|s|\right)\right]
\end{aligned}
$$

where the asterisk $\left({ }^{*}\right)$ denotes complex conjugation and $E_{1}(z)$ is the exponential integral. ${ }^{23}$ In these expressions the complex wave number $k_{c}$ is defined as $k_{c}=k+i \nu$, where $k>0$ represents the carrier wave number of the excited MSSW. It can be calculated as the root of the approximate dispersion relation

$$
W(k)[W(k)+1]+\operatorname{Re}\left[\frac{\chi+1}{\chi_{a}^{2}-\chi^{2}}\right]=0,
$$

where $W(k)=[\exp (-|k| L)-1] /(|k| L)$ and $\chi$ and $\chi_{a}$ are the diagonal and off-diagonal components of the microwave magnetic-susceptibility tensor $\hat{\chi}$ for the film material. ${ }^{17}$ The tensor components are complex numbers, as magnetic losses in the material are included.

The imaginary part of $k_{c}$ determines the spatial spin-wave damping and is negative $(\nu \leq 0)$ for MSSWs:

$$
\nu=-[D(k)]^{-1} \operatorname{Im}\left[\frac{\chi+1}{\chi_{a}^{2}-\chi^{2}}\right],
$$

where

$$
D(k)=\frac{d}{d(|k|)}\{W(k)[W(k)+1]\}<0 .
$$

The negative value of $\nu$ shows that the direction in which the wave amplitude decreases due to damping coincides with the direction in which the wave phase $\phi(y)$ increases [see Eq. (8)]. The wave is therefore a forward one which is consistent for MSSWs (for comparison, for BMSWs $\nu>0$ and the waves are backward ones ${ }^{20}$ ).

As one sees from Eqs. (5)-(8), the scalar function $F(s)$ determines the phase accumulation while the matrix-vector product $\frac{1}{D(k)} \hat{Y} \cdot \boldsymbol{h}_{s}$ determines the MSSW initial phase and hence is responsible for the excitation phase. Indeed, like in the BVMSW case ${ }^{20}$ the near (unretarded) field of the antenna is proportional to the terms in Eq. (8) involving the exponential integrals $E_{1}(s)$ the sum of which is real. Far away from the antenna the near field vanishes and the $y$ dependence of the dynamic magnetization is determined by the first term in the brackets in Eq. (8) which is an intrinsically complex function of $s$ and is purely imaginary at the source point $s=0$. This term describes a retarded field (traveling waves) propagating in both directions from the excitation source:

$$
F(s)=-i \exp (-i|k s|) .
$$

(In this formula and onwards for the sake of simplicity we ignore magnetic losses by setting $\nu=0$.) In contrast to Eq. (4) the sign of the exponent is negative. This results in a positive slope for the phase accumulation $\phi(y)=|k y|$ for MSSWs as demonstrated by our experiments.

All the expressions above are valid for long-wavelength MSSW with carrier wave vectors $k$ for which $W(k)<-0.5$. For very small wave vectors $(k L \ll 1)$ one can use the ap- proximation $W(k) \approx-1+(|k| L) / 2, D(k) \approx L / 2=D$. Furthermore, for $\nu=0, \chi$ and $\chi_{a}$ are real and for $k L \ll 1$ from the dispersion relation Eq. (9) one obtains $k=2(\chi+1) /\left[L\left(\chi_{a}^{2}\right.\right.$ $\left.\left.-\chi^{2}\right)\right]$. Then the elements of the matrix $\hat{S}=\hat{Y} / D$ reduce to

$$
\begin{gathered}
S_{11}=-\frac{2}{L\left(\chi_{a}^{2}-\chi^{2}\right)}, \\
S_{12}=\frac{2 i \chi_{a}}{L\left(\chi_{a}^{2}-\chi^{2}\right)}, \\
S_{21}=-\frac{2 i \chi_{a}}{L\left(\chi_{a}^{2}-\chi^{2}\right)}, \\
S_{22}=\frac{2\left[(\chi+1)^{2}-\chi_{a}^{2}\right]}{L\left(\chi_{a}^{2}-\chi^{2}\right)} .
\end{gathered}
$$
into

Using Eqs. (6), (12), and (13), Eq. (5) is then transformed into

$$
\boldsymbol{m}( \pm|y|)=-2 \pi i \hat{S} \exp (-i|k y|) \boldsymbol{h}_{ \pm|k| s},
$$

where $\boldsymbol{h}_{k s}=(2 \pi)^{-1} \int_{-\infty}^{\infty} \boldsymbol{h}_{s}(y) \exp (i k y) d y$ is the spatial Fourier transform of the antenna field. From this formula one sees that the initial phases of the excited waves are determined by the phase of the product $\hat{S} \boldsymbol{h}_{ \pm|k| s}$.

As discussed in the previous section, the Fourier transform of the out-of-plane component of the antenna field is an odd function of $k$. On the contrary, since the spatial profile of the in-plane component $h_{s y}(y)$ is symmetric with respect to the longitudinal antenna axis $y=0$, its Fourier transform is an even function and thus $h_{-|k| s y}=h_{|k| s y}$. Furthermore, from Maxwell equations one finds that for a microwave field generated by a current through a microstrip transducer it stands $h_{k s x}=i \operatorname{sign}(k) h_{k s y}$ [see e.g., Eq. (32) in Ref. 24]. Accounting for the change in sign in Eq. (14) this formula can be written as $h_{k s x}=i \operatorname{sign}(y) h_{k s y}$ and the vector $\boldsymbol{h}_{ \pm|k| s}$ in Eq. (14) takes the form

$$
\boldsymbol{h}_{ \pm|k| s}=\left(\begin{array}{c}
i \operatorname{sign}(y) \\
1
\end{array}\right) h_{|k| s y} .
$$

One now sees that the MSSW initial phase is determined by the product of the matrix $\hat{S}$ with the vector $[i \operatorname{sign}(y), 1]^{T}$.

It is sufficient to consider only the first element of the resulting vector (the second one differs from the first one only by the coefficient of ellipticity of the precession of the magnetization vector and by the phase shift of $\pi / 2$ ). Using Eqs. (14) and (15), $m_{x}$ can be written as

$$
m_{x}( \pm|y|)=\frac{4 \pi}{L} h_{|k| s y} \frac{\chi_{a}-\operatorname{sign}(y)}{\chi_{a}^{2}-\chi^{2}} \exp (-i|k y|) .
$$

As in our range of frequencies and static fields $\chi_{a}<-1$, the real coefficient in front of the complex exponent in Eq. (16) has the same sign for positive and negative $y$ values, i.e., for the positive and the negative waves excited by the same antenna. This shows that the MSSW excitation phase is zero, as confirmed by our measurements. 


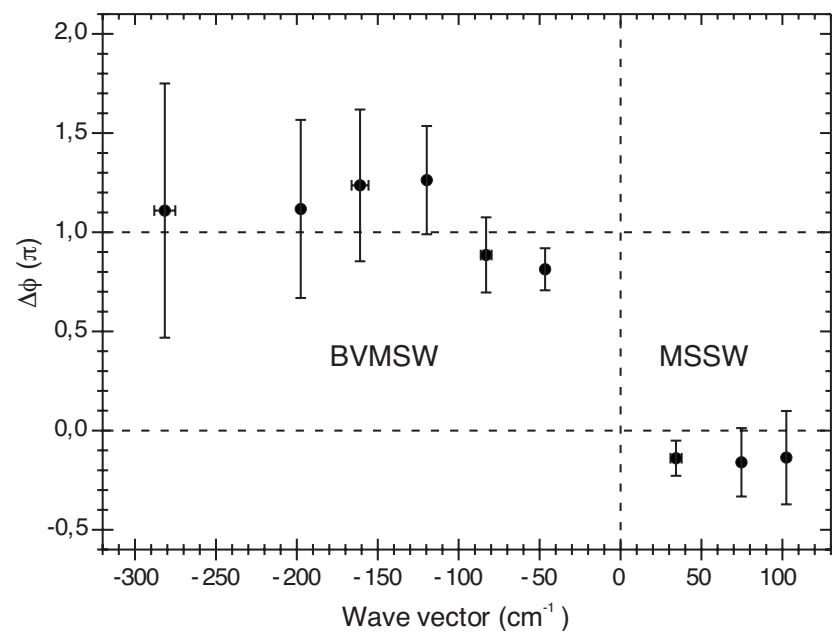

FIG. 4. Summary of the performed measurements. The expected behavior is clearly visible. The different excitation phase values for the lowest absolute values of the wave vectors can be explained by the distortion due to the propagation close to the ferromagnetic resonance frequency.

\section{SUMMARY AND CONCLUSION}

Figure 4 shows a summary of all performed experiments.
The above-described behavior is clearly visible for all wave vectors. The notably different excitation phase for the measurements with the lowest absolute value for the wave vector can be explained by taking into account that a spin-wave pulse propagating close to the ferromagnetic resonance frequency becomes strongly distorted. This distortion also influences the phase and thus the measured values for the excitation phase. Note that the main source of error is the position of the antenna. Since this position error translates to the phase error as $k \cdot \Delta x$ the error margins become larger for larger wave vectors.

In conclusion we have demonstrated that two counterpropagating backward volume waves which are excited by the microstrip transducer symmetrically in amplitude show an excitation phase of $\pi$ while the nonreciprocal surface waves which are unsymmetrical in amplitude have zero excitation phase.

\section{ACKNOWLEDGMENTS}

Financial support by the Deutsche Forschungsgemeinschaft (Graduiertenkolleg 792 and MATCOR) and the Australian Research Council and technical support from the Nano+Bio Center, TU Kaiserslautern are acknowledged. *tschneider@physik.uni-kl.de

$\dagger$ On leave from St. Petersburg Electrotechnical University, St. Petersburg, Russia; kostylev@ cyllene.uwa.edu.au

${ }^{1}$ R. W. Damon and J. R. Eshbach, J. Phys. Chem. Solids 19, 308 (1961).

${ }^{2}$ G. A. Melkov, V. I. Vasyuchka, V. V. Lazovskiy, V. S. Tiberkevich, and A. N. Slavin, Appl. Phys. Lett. 89, 252510 (2006).

${ }^{3}$ G. A. Melkov, V. I. Vasyuchka, A. V. Chumak, V. S. Tiberkevich, and A. N. Slavin, J. Appl. Phys. 99, 08P513 (2006).

${ }^{4}$ A. N. Slavin, O. Büttner, M. Bauer, S. O. Demokritov, B. Hillebrands, M. P. Kostylev, B. A. Kalinikos, V. V. Grimalsky, and Yu. Rapoport, Chaos 13, 693 (2003).

${ }^{5}$ M. P. Kostylev and N. G. Kovshikov, Tech. Phys. 47, 1350 (2002).

${ }^{6}$ N. G. Kovshikov, B. A. Kalinikos, C. E. Patton, E. S. Wright, and J. M. Nash, Phys. Rev. B 54, 15210 (1996).

${ }^{7}$ M. Chen, M. A. Tsankov, J. M. Nash, and C. E. Patton, Phys. Rev. Lett. 70, 1707 (1993).

${ }^{8}$ W. E. Kunz, K. W. Chang, and W. Ishak, Proc.-IEEE Ultrason. Symp., 187 (1986).

${ }^{9}$ T. D. Poston and D. D. Stancil, J. Appl. Phys. 55, 2521 (1984).

${ }^{10}$ M. P. Kostylev, V. E. Demidov, U.-H. Hansen, and S. O. Demokritov, Phys. Rev. B 76, 224414 (2007).

${ }^{11}$ M. P. Kostylev, A. A. Serga, T. Schneider, B. Leven, and B. Hillebrands, Appl. Phys. Lett. 87, 153501 (2005).

${ }^{12}$ T. Schneider, A. A. Serga, B. Leven, B. Hillebrands, R. L.
Stamps, and M. P. Kostylev, Appl. Phys. Lett. 92, 022505 (2008).

${ }^{13}$ T. Schneider, A. Serga, B. Hillebrands, and M. Kostylev, J. Nanoelectron. Optoelectron. 3, 69 (2008).

${ }^{14}$ A. A. Serga, T. Schneider, B. Hillebrands, S. O. Demokritov, and M. P. Kostylev, Appl. Phys. Lett. 89, 063506 (2006).

${ }^{15}$ O. Büttner, M. Bauer, S. O. Demokritov, B. Hillebrands, Y. S. Kivshar, V. Grimalsky, Yu. Rapoport, and A. N. Slavin, Phys. Rev. B 61, 11576 (2000).

${ }^{16}$ T. Schneider, A. A. Serga, B. Hillebrands, and M. P. Kostylev, Europhys. Lett. 77, 57002 (2007).

${ }^{17}$ A. G. Gurevich and G. A. Melkov, Magnetization Oscillations and Waves (CRC, New York, 1996).

${ }^{18}$ K. Yu. Guslienko, S. O. Demokritov, B. Hillebrands, and A. N. Slavin, Phys. Rev. B 66, 132402 (2002).

${ }^{19}$ M. P. Kostylev and N. A. Sergeeva, in Magnetic Properties of Laterally Confined Nanometric Structures, edited by G. Gubbiotti (Transworld Research Network, Kerala, India, 2006).

${ }^{20}$ M. P. Kostylev, A. A. Serga, T. Schneider, T. Neumann, B. Leven, B. Hillebrands, and R. L. Stamps, Phys. Rev. B 76, 184419 (2007).

${ }^{21}$ M. P. Kostylev, G. Gubbiotti, J.-G. Hu, G. Carlotti, T. Ono, and R. L. Stamps, Phys. Rev. B 76, 054422 (2007).

${ }^{22}$ B. A. Kalinikos, Sov. Phys. J. 24, 718 (1981).

${ }^{23}$ Handbook of Mathematical Functions, edited by N. Abramovitz and I. Stegun (Dover Publications, New York, 1965).

${ }^{24}$ V. F. Dmitriev and B. A. Kalinikos, Sov. Phys. J. 31, 875 (1988). 